\title{
La Ley marroquí de violencia contra las mujeres: una aproximación al contenido y al debate ideológico
}

\section{The Moroccan Law on Violence against Women: an Approach to its Content and the Ideological Debate}

\author{
Carmelo Pérez Beltrán ${ }^{1}$ \\ Universidad de Granada (España) \\ ORCID: https://orcid.org/0000-0002-0108-8863
}

Recibido: 15-12-2020

Aceptado: $27-12-2020$

\section{Resumen}

Este artículo tiene un doble objetivo: en primer lugar, estudiar las principales aportaciones y carencias de la Ley $\mathrm{n}^{\circ} 103.13$ referente a la lucha de la violencia contra las mujeres, que fue promulgada en Marruecos el 12 en marzo de 2018. En segundo lugar, analizar los principales puntos de tensión política e ideológica que existen entre la sociedad civil más comprometida con la violencia de género en Marruecos, cuyos referentes beben de las declaraciones y convenciones internacionales, y las instituciones gubernamentales del Partido de la Justicia y el Desarrollo, en el poder desde enero de 2012, cuyos planteamientos se encuentran

\footnotetext{
${ }^{1}$ (carmelop@ugr.es). Catedrático de Estudios Árabes e Islámicos en la Universidad de Granada, Director de la Cátedra Emilio García Gómez y Director de la revista Miscelánea de Estudios Árabes y Hebraicos. Sección Árabe-Islam de esta misma Universidad. Coordinador del Grupo de Investigación Estudios Árabes Contemporáneos. Especializado en Sociología del Mundo Árabe, sus principales líneas de investigación son cuatro: estudio de las mujeres y el género (Mujeres argelinas en la lucha por las libertades democráticas, Ganada: Editorial Universidad de Granada, 1997, entre otros), sociedad civil y transición política en el Magreb (Sociedad civil, derechos humanos y democracia en Marruecos, Granada: Editorial Universidad de Granada, 2006, entre otros), derechos humanos y paz en contexto árabe ("Religión y derechos humanos: la visión del islam", en J. Morandé y C. Pomerleau, eds., Globalización y visiones religiosas. Opciones por los derechos humanos y el medio ambiente, Santiago de Chile: Ril Editores, 2002, 115-127) y cambios jurídicos en el Magreb ("Pluralismo bajo control: evolución de la Ley de partidos políticos en Argelia", Estudios de Asia y África, 52, 2 (2017), pp. 31- 60, entre otros).
} 
determinados por la ideología islamista y por ciertas especificidades simbólicoreligiosas del islam.

Palabras-clave: Violencia de género, Marruecos, violencia contra las mujeres, legislación.

\begin{abstract}
This article has two objectives: firstly, studying the main contributions and deficiencies of the Law No. 103.13 on the fight of violence against women, enacted in Morocco on March 12th 2018. Secondly, studying ideological stress points between the civil society that is most committed with gender violence in Morocco, whose referents are based on the international declarations and conventions, and the governmental institutions of the Justice and Development Party, in power from January 2012, whose approaches are determined by the Islamist ideology and by certain symbolic-religious Islamic specialties.
\end{abstract}

Keywords: Gender violence, Morocco, violence against women, legislation.

\title{
1. Introducción
}

El 12 de marzo de 2018 fue publicado en el Boletín Oficial del Reino de Marruecos, en su versión árabe oficial, el Dahir 1.18.19 con la promulgación de la Ley $n^{\circ} 103.13$ referente a la lucha de la violencia contra las mujeres (A1Ŷāida al-Rasmiyya 2018: 1449-1455), cuya entrada en vigor comenzó seis meses después (art. 18), es decir, el día 12 de septiembre de 2018. Resulta significativo incidir en la cuestión de la "versión oficial" por varios motivos; entre ellos, por el hecho de que la traducción "oficiosa" en lengua francesa no fue publicada hasta cuatro meses después, el 5 de julio de 21018 (Bulletin Officiel 2018: 1384-1389), lo cual no es lo usual en Marruecos², y nos informa ya del conflicto ideológico que presupone este tema.

Tampoco podemos decir que la adopción de esta Ley surja por "generación espontánea", sino que detrás de esta norma existe una dura realidad social (Pérez 2020: 343-353) que, de forma objetiva, había sido detectada por la

\footnotetext{
${ }^{2}$ Por regla general, el Boletín Oficial del Reino de Marruecos (BORM) se publica dos veces a la semana, los lunes y los jueves, en su versión oficial en lengua árabe. Por su parte, la versión francesa es bimensual y se publica el primer y el tercer jueves de cada mes. Mientras que el BORM en árabe incluye, además de las leyes y todo tipo de normas nacionales, los debates parlamentarios y anuncios de diversa naturaleza, por su parte la versión en lengua francesa solamente incide en el primer aspecto señalado, leyes y normas (Benabdallah 2012: 359-363).
} 
Enquête nationale sur la prévalence de la violence à l'égard des femmes ${ }^{3}$, realizada entre julio de 2009 y enero de 2010, bajo el paraguas institucional del Alto Comisariado del Plan, con el apoyo de organismos internacionales como ONU-Mujeres y el Fondo para el logro de los Objetivos de Desarrollo del Milenio. Basada en una muestra de 8.300 mujeres de 18 a 65 años, esta encuesta revelaba que el $62,8 \%$ de las mujeres marroquíes (es decir, casi 6 millones sobre un total de 9,5 millones de mujeres) de edad comprendida entre los 18 y los 64 años había sufrido algún acto de violencia en el último año, especialmente violencia psicológica $(48,4 \%)$, seguida de atentados a la libertad $(31,3 \%)$, violencia relacionada con la infracción de las leyes $(17,3 \%)$, violencia física $(15,2 \%) \mathrm{y}$, finalmente, violencia sexual $(8,7 \%)$ (Haut-Commissariat au Plan 2012). Un poco más cercano en el tiempo, en 2019, el Rapport sur les violences faites aux femmes et aux filles, llegaba a la conclusión de que una de cada dos mujeres marroquíes había sufrido algún tipo de violencia durante los últimos doce meses, lo cual equivaldría a una tasa de prevalencia del $57 \%$ a nivel nacional (Haut-Commissariat au Plan 2019: 26).

A pesar de estos datos sociológicos preocupantes, es necesario señalar que uno de los grandes déficits del país en materia de violencia de género es la ausencia de indicadores nacionales unificados y fiables, de tal manera que las cifras cuantitativas que baraja el país provienen principalmente cuatros organismos oficiales que aportan datos a partir de las denuncias o gestiones de las víctimas, pero de forma autónoma y sin coordinación entre ellos: el Ministerio de Justicia, el Ministerio de Salud, la Dirección General de Seguridad Nacional y la Gendarmería Real. Por poner algún ejemplo y tomando como referencia el Ministerio de Justicia, queda patente que la violencia contra las mujeres es un fenómeno en aumento constante, puesto que el número de denuncias que se han ido registrando por este motivo no ha cesado de aumentar en los últimos años, pasando de 11.30 en 2013 a 16.690 en 2017 (Pérez 2020: 345-347). Un poco más reciente en el tiempo, durante los años 2018-2019 se han registrado una media de 1.500 denuncias mensuales (MAP 2020), es decir, unas 18.000 al año. El debate sobre la incidencia de la violencia de género en el país se ha vuelto a abrir durante los recientes meses de confinamiento debido a la pandemia del COVID, a partir de marzo de 2020, ya que han surgido voces desde la sociedad civil denunciando un aumento preocupante a consecuencia del aislamiento de las mujeres en su entorno familiar. Frente a esta percepción, el Procurador General del Rey, Mohamed Abdennabaoui, hizo unas declaraciones en mayo de 2020 con la intención de demostrar que durante el periodo de confinamiento el número de denuncias por violencia de género, contrariamente a lo que se estaba especulando, había descendido considerablemente, pasando de una media

\footnotetext{
${ }^{3}$ Sus resultados no fueron publicados hasta el año 2012, es decir, dos años después de la realización de la encuesta y un año después del estallido de la Primavera Árabe.
}

Araucaria. Revista Iberoamericana de Filosofí, Política, Humanidades y Relaciones Internacionales, año $23, \mathrm{n}^{\circ} 48$. Tercer cuatrimestre de 2021. Pp. 165-185. ISSN 1575-6823 e-ISSN 2340-2199 https://dx.doi.org/10.12795/araucaria.2021.i48.08 
mensual de 1.500 denuncias a 148 durante la primera mitad del 2020, es decir, diez veces menos. Sin embargo, estos datos han sido cuestionados por diversas asociaciones del país (Lefébure 2020) que defienden una lectura distinta a la oficial, puesto que el descenso del número de casos se encuentra relacionado, más bien, con la limitación del movimiento, propio de estos meses, y con la obligatoriedad de interponer denuncias o comunicar casos por vía electrónica, exclusivamente.

Debido a la situación descrita anteriormente, al menos desde los años 90 del pasado siglo, surgió todo un movimiento organizado que hizo de este tipo de violencia una de sus principales señas de identidad, tomando como referente teórico e institucional la Declaración sobre la Eliminación de la Violencia contra la Mujer (DEVM), aprobada por la Asamblea General de las Naciones Unidas en 1993. De esta forma, en 1995 surgió la Asociación Marroqui de Lucha contra la Violencia contra las Mujeres ${ }^{4}$, pero también las principales asociaciones feministas del país ${ }^{5}$, con más voluntad que recursos, se implicaron en diferentes programas de lucha y sensibilización, y crearon infraestructuras de apoyo a las víctimas y a sus hijos, incluyendo centros de atención y de asistencia jurídica, médica o psicológica, casas de acogida, teléfonos de escucha, informes y tratamiento de datos estadísticos, etc.

Tampoco la acción gubernamental ha quedado al margen de esta lucha y desde 1998 el gobierno marroquí ha puesto en marcha campañas anuales de sensibilización (Chikhaoui 2008), así como diferentes estrategias y programas nacionales (Secrétariat d'Etat Chargé de la Famille 2015), en colaboración con organismos internacionales y otras instancias extranjeras. Entre ellos, caben destacar la Estrategia Nacional de Lucha contra Violencia contra las Mujeres, presentada oficialmente en marzo de 2002, y sobre todo, el Programa multisectorial de lucha contra las violencias de género-Tamkine ${ }^{6}$ (2008-2011) que, con una financiación de 84 millones de dólares (Dialmy 2012, 46), tenía por objetivo principal la institucionalización y territorialización de la lucha contra la violencia de género mediante la coordinación de diferentes departamentos ministeriales junto a varias agencias internacionales de Naciones Unidas ${ }^{7}$ y la sociedad civil del país, en el marco de los Objetivos de Desarrollo del Milenio (ODM). También es cierto que esta acción gubernamental ha sido considerada por los agentes sociales como insuficiente e ineficaz, debido tanto a la escasez

\footnotetext{
${ }^{4}$ Su web puede consultarse en http://amvef.org [13-10-2021].

5 Entre otras asociaciones: Unión de Acción Femenina, Asociación Democrática de Mujeres de Marruecos, Liga de Defensa de los Derechos de las Mujeres, Iniciativas Femeninas, Aspiraciones Femeninas y Jossour (Olmedo 2006: 281-283; Dialmy 2012: 51-53).

${ }^{6}$ En árabe tamkīn significa consolidación, intensificación, capacitación. Los diferentes documentos de este programa (estudios, guías y evaluaciones) pueden ser consultados en la web oficial http:// www.social.gov.ma/fr/femme/programme-tamkine [13-10-2021].

${ }^{7}$ Principalmente las siguientes: UNIFEM, UNFPA, UNICEF, BIT, FAO, UNESCO, UNHCR, NUSIDA, UNIFEM.
} 
de los recursos económicos y humanos destinados a este fin, como a la falta de una verdadera voluntad política para ejecutar, de forma decidida, los objetivos marcados en las diferentes campañas, estrategias y programas. En este sentido, un informe del Consejo económico, social y medioambiental del año 2015 afirmaba:

El presupuesto asignado al Ministerio de Solidaridad, Mujer y Familia para el año 2015 fue de 649 millones de dírhams para todas sus acciones, de los cuales solamente un sexto (algo más de 100 millones) estaba destinado a inversiones. Solo el 5,26\% de esta cantidad se dedicó explícitamente a la promoción de los derechos de la mujer, incluida la acción para la institucionalización y difusión de los principios de equidad e igualdad, la lucha contra todas las formas de discriminación, violencia y exclusión, y la promoción de la igualdad de oportunidades. En 2015, este Ministerio contaba solamente con 387 empleados, mientras que, por ejemplo, el Ministerio de Cultura contaba con 1.761 agentes y el Ministerio de Trabajo con 1.441. Su presupuesto para inversiones, destinado a cubrir el apoyo a la infancia, las familias, las mujeres víctimas de violencia y las personas con discapacidad, así como a la protección y promoción de los derechos de la mujer, era apenas superior al ya de por sí exiguo asignado al Ministerio de Trabajo (86 millones de dírhams), y muy inferior a los 180 millones de dírhams del Ministerio de Cultura (Conseil Économique, Social et Environnemental 2006: 23-24).

Ello es especialmente significativo si tenemos en cuenta que desde los años 90 del pasado siglo XX Marruecos ha adquirido una serie de compromisos internacionales de gran relevancia, especialmente la Convención para la Eliminación de todas las Formas de Discriminación contra la Mujer (CEDAW), ratificado en 1993 con reservas, hasta que fueron levantadas en 2011, y la Plataforma de Acción derivada de la IV Conferencia Mundial sobre las Mujeres (Beijing, 1995), entre otros.

A ello se añade la fragmentación del debate ideológico entre dos modelos de sociedad que parecen irreconciliables, especialmente cuando se incide en temas como el estatus de las mujeres, el modelo familiar, las identidades de género o las orientaciones sexuales (Pérez 2020: 347-350). Dicho debate, en el que profundizaremos más adelante, toma mayores dimensiones a partir del desarrollo y consolidación del islamismo en el país, cuyo partido hegemónico, el Partido de la Justicia y el Desarrollo (PJD), fue aumentando progresivamente su representación parlamentaria hasta llegar al éxito electoral de noviembre de 2011, que le permitió asumir el gobierno del país hasta las últimas elecciones legislativas, celebradas en septiembre de 2021 (Desrues y Fernández 2013; Desrues y Hernando de Larramendi 2011; Parejo 2010; White 2008). Será en determinados momentos puntuales cuando este debate, caracterizado por el antagonismo entre los defensores de las especificidades culturales del 
islam frente a los partidarios de los referentes universales, alcanza mayores dimensiones, tal y como ocurrirá con la adopción de la Ley $\mathrm{n}^{\mathrm{o}} 103.13$ referente a la lucha de la violencia contra las mujeres, en marzo de 2018.

Teniendo en cuenta los planteamientos citados anteriormente, este trabajo, además de señalar las aportaciones más importantes de esta Ley, tiene por objetivo estudiar los principales puntos de tensión ideológica entre la sociedad civil más comprometida con esta cuestión, cuyos referentes beben de las declaraciones y convenciones internacionales que versan sobre este tema, y las instituciones gubernamentales del islamista Partido de la Justicia y el Desarrollo, en el poder desde enero de 2012 hasta septiembre de 2021, cuyos planteamientos se encuentran determinados por ciertas especificidades jurídicas y religiosas del islam (Macías 2005: 112-114). Como fruto de esta polarización ideológica, la Ley 103.13 será calificada por la primera tendencia señalada como una norma débil, insuficiente, cosmética o conservadora, mientras que la segunda la definirá como uno de los textos más importantes en el dominio de la igualdad de los sexos.

\section{Hacia la adopción de una Ley de violencia contra las mujeres}

Hasta la promulgación de la Ley 103-13, la violencia de género ha estado jurídicamente regulada a través de las disposiciones del Código Penal, cuya principal reforma, en este sentido, data de 2003 (Jeddi 2015: 1007-1033.). Entre otras medidas, esta reforma reconocía como delito la discriminación por razón de sexo, la violencia entre los cónyuges (art. 404) ${ }^{8}$, el acoso sexual (art. 503) y el secuestro y tráfico de personas. Sin embargo, otras prescripciones, como el artículo 475, que facilitaba la impunidad a los violadores de niñas menores de edad si contraían matrimonio con la víctima, siguió en vigor hasta que fue definitivamente modificado en febrero de 2014 (Bulletin Officiel 2014), debido al gran debate generado en el país tras el suicidio en 2012 de la joven Amina Filali, de 16 años, obligada a casarse con su violador y que, tras el matrimonio, había sido víctima de violencia familiar (Nima 2018: 49-59). Otras cuestiones perjudiciales para las mujeres víctimas de la violencia siguen aún vigentes en el Código Penal, debido a la pervivencia de un modelo familiar impregnado de valores religiosos, en donde prevalece la autoridad del marido sobre los derechos fundamentales de las mujeres, como es el caso del artículo 496 que castiga a las personas que oculten a las mujeres casadas. Según la Asociación Marroqui de Lucha contra la Violencia contra las Mujeres, este artículo supone

\footnotetext{
${ }^{8}$ El artículo en cuestión habla de "violencia entre los cónyuges", sin precisar el caso particular de la violencia contra la esposa. Además no contemplaba los casos de violencia cometidos por parejas no casadas, novios o exparejas.
} 
una preponderancia de la institución familiar sobre la propia supervivencia de las mujeres y "constituye un problema, por no decir un obstáculo, al desarrollo de centros de acogida o de refugios cuya función principal es ocultar a las mujeres que huyen" (AMVEF 2010: 16).

En este sentido es necesario tener en cuenta que para el ideario político del PJD, la familia, en tanto que base de la sociedad y garantía para el desarrollo del país, debe estar sustentada y protegida por la $\check{s} \operatorname{rr}^{\circ}{ }^{`} a$, la ley islámica, puesto que "es la garantía de la realización humana más noble, la misericordia y la justicia, por lo que su respeto y su cuidado son fundamentales (implícitamente se sitúa el papel de la mujer como madre y esposa)" (Macías 2005: 113). En definitiva, como afirma la Asociación Marroquí de Mujeres Victimas de Violencia, "aunque las discriminaciones más fragrantes hayan sido suprimidas del Código Penal, su espíritu, basado en la dominación del grupo familiar sobre el individuo, no ha cambiado" (Zirari 2007: 15), además de que las cuestiones relacionadas con violencia de género se encontraban dispersas e inconexas dentro de un complejo entramado jurídico, muy influenciado por la moral islámica.

Como consecuencia de la trayectoria acumulada durante años y del nuevo clima de cambios tras la Primavera Árabe, el Anteproyecto de Ley 103-13 fue sometido al Consejo de Gobierno, presidido por el líder del PJD de aquella época, Abdelilah Benkirán, en noviembre de 2013, en medio de un clima bastante crispado, debido al malestar suscitado entre las asociaciones de la sociedad civil más relacionadas con la violencia de género, que acusaban al gobierno de no haber contado con sus reivindicaciones y su larga experiencia acumulada durante décadas. No menos encrespadas eran las críticas de otros sectores sociales y políticos, dentro incluso del PJD, que acusaban al texto de ser un producto de la tutela laica occidental, cuyos dictámenes pretenden introducir en el país sus problemas, sus planteamientos y sus fondos económicos, como resultado de una política neocolonial que atenta contra la identidad nacional de Marruecos y contra los valores de la familia musulmana?.

Debido a este clima hostil, la adopción de este Anteproyecto de Ley fue pospuesta y, como solución inmediata, el Presidente del Gobierno nombró una comisión encargada de analizar y revisar el texto bajo la responsabilidad de dos ministerios: el de Justicia y Libertades y el de Solidaridad, Mujer, Familia y Desarrollo Social, encabezado por Basima Haqawi. Después de tres años de

\footnotetext{
${ }^{9}$ Algo similar había ocurrido en el año 2000 cuando el gobierno de 'Abdelrraḥmān Yūsufī, (19982002) pretendió adoptar un Plan de Acción Nacional para la Integración de la Mujer al Desarrollo. En esta ocasión las tendencias islamo-islamista de Marruecos concibieron dicho Plan de Acción como un gravísimo atentado a la identidad musulmana del país, como una invasión neo-imperialista y como una especie de complot mundial contra el islam. Incluso el Ministro de Asuntos Islámicos dijo de él que "en su enfoque y su contenido, se trata de una de las facetas de la guerra civilizacional contra el renacimiento islámico", y el Comité Nacional para la Protección de la Familia aseguró: "estamos ante un plan mundial que pretende imponer el modelo occidental en el sistema social y familiar marroquí" (Pérez y Macías 2017: 15-22).
} 
estudio por parte de esta Comisión y dos años más de tramitaciones, finalmente el Ley 103-13 fue aprobada por el Parlamento el 14 de febrero de 2018, en medio de un clima general de apatía y con escasa presencia de parlamentarios (Lefébure 2016) y publicada en el Boletín Oficial el 12 de marzo (versión árabe). También es cierto que durante todos estos años se formó todo un lobby de organizaciones de defensa de las mujeres ${ }^{10}$ y de derechos humanos con diferentes propuestas y recomendaciones para exigir mejoras en un texto que consideraban poco ambicioso y demasiado influenciado por la moral islámica. Entre ellas, posiblemente la más importante fue la que presentó el Conseil National des Droits de l'Homme (2018), difundida por diferentes medios.

\section{Aproximación a la Ley $n^{0} \mathbf{1 0 3 . 1 3}$ referente a la lucha de la violencia contra las mujeres}

La Ley $103.13^{11}$ se encuentra estructurada en seis capítulos destinados a definir la violencia contra las mujeres y sus tipos (Capítulo 1), modificar y/o completar algunas normas del Código Penal (Capítulo 2) o del Código de Procedimiento Penal (Capítulo 3) y establecer mecanismos de apoyo a las mujeres víctimas (Capítulo 4). Por su parte el Capítulo 5, compuesto por un único artículo (art. 17), compromete a las autoridades públicas a poner en marcha medidas de sensibilización y prevención, mientras que el último Capítulo establece la entrada en vigor de la ley.

Definida la violencia contra "la mujer" (escrito en singular, al-mar'a, en el art. 1 de la Ley en su versión oficial en árabe y en plural, femmes, en la francesa) $)^{12}$ como "cualquier acto material, moral o impedimento basado en la discriminación de sexo con resultado de daño corporal, psicológico, sexual o económico para la mujer", la mayor parte del artículo primero tiene por finalidad distinguir y definir cuatro formas o manifestaciones diferentes: la violencia corporal (cualquier acto o impedimento que suponga o pueda suponer un perjuicio para la integridad física de la mujer), la violencia sexual (cualquier palabra, acto o explotación con fines sexuales o comerciales que pueda suponer un atentado a la integridad corporal de la mujer), violencia psicológica (cualquier agresión verbal, amenaza, negligencia o privación

${ }^{10}$ Entre ellas, la Coalición Primavera por la Dignidad, creada en el año 2010, en la que participaron unas 25 asociaciones. Su blog: https://pdmaroc.wordpress.com/qui-sommes-nous/ [13-10-2021].

11 Versión árabe (oficial): Al-Ŷ́arīda al-Rasmiyya (2018). Versión francesa: Bulletin Officiel (2018).

12 La cuestión del número gramatical (singular/plural) traduce también ciertos conflictos entre referencias ideológicas exógenas y endógenas. El término plural "mujeres", muy relacionado con el desarrollo de los feminismos periféricos, es reivindicado por el feminismo occidental desde los años 80 y hace referencia, según estos planteamientos, a los sujetos históricos y a la heterogeneidad de vivencias entre las propias mujeres. En cambio, el empleo del singular se encuentra más relacionado con la especificidad de la mujer musulmana y con la identidad nacional marroquí. 
contra la dignidad, la libertad y la tranquilidad de la mujer, o con el objeto de intimidarla o aterrorizarla), y la violencia económica (cualquier acto o impedimento de naturaleza económica o financiera que afecte o pueda afectar los derechos sociales o económicos de la mujer). En general, la definición se aproxima a la DEVM, aunque resulta significativo que entre las formas de violencia no se haga una referencia específica a la violencia conyugal que, según había revelado la Enquête Nationale, anteriormente señalada, constituye la mayor prevalencia de la violencia de género. Cuestión ésta relacionada, sin duda, con la moral islámica en torno a la familia que propone el PJD, como señalamos anteriormente.

En materia penal, uno de los principales avances de la Ley 103.13 consiste en el aumento significativo de las penas (generalmente el doble con respecto a la legislación anterior) en tres casos particulares: en primer lugar en el caso de violencia contra la mujer "por razón de sexo" (art. 404) o lo que podríamos llamar "violencia sexista"; en árabe el término empleado de manera reiterativa a lo largo de todo la Ley es bi-sabab yinsi-ha (por razón de su sexo), que es el que utilizaremos en este estudio para aproximarnos mejor al contenido de la norma. En segundo lugar, en el supuesto de que la violencia, la falta de asistencia, rapto o secuestro hayan sido cometidos por el marido, exmarido, novio, un ascendiente, un descendiente, un hermano o un tutor. Finalmente, en el caso de que la víctima sea menor de edad o tenga alguna discapacidad (art. 431).

Igualmente, la Ley 103.13 introduce una serie de medidas de protección de las víctimas, sometidas siempre a la consideración del tribunal competente, entre las cuales destaca la prohibición del hombre condenado por violencia de género de contactar con la víctima, acercarse o comunicarse con ella por cualquier medio durante un período máximo de cinco años (art. 88.1). La violación de dicha medida podría conllevar una pena de cárcel de dos meses a seis años y/o una multa de 2.000 a 20.000 dirhams. Sin embargo, asociaciones de derechos humanos, como Human Rights Watch (2018) han puesto en evidencia las limitaciones de esta norma, teniendo en cuenta que medidas cautelares como la orden de alejamiento, la prohibición de comunicación o la prohibición de residir en determinados lugares, entre otras, pueden ser determinantes para prevenir nuevas formas de violencia $\mathrm{o}$, incluso, para salvar vidas en riesgo inminente. En este sentido, el principal escollo reside en el hecho de que la Ley 103.13 solo prevé medidas cautelares en el contexto de un proceso judicial o después de que el hombre haya sido condenado, mientras que la agencias internacionales (ONU Mujeres 2010: 48-51) recomiendan que las mujeres puedan solicitar órdenes de protección de urgencia mediante procedimiento civil e independientemente de cualquier otro procedimiento. Peor aún, la Ley 103.13 no prevé recursos de acogida 
(casas de acogida, centros de emergencias, pisos tutelados, etc.) para proteger y alojar a las mujeres víctimas de violencia y sus hijos, que se ven en la necesidad de abandonar el domicilio conyugal para preservar su integridad física o psicológica. Generalmente, este tipo de recursos los vienen ofreciendo algunas asociaciones, aunque en su conjunto no superan la decena de centros a nivel nacional (AMVEF 2010: 5). Como señalamos anteriormente, para el ideario político-religioso del PJD, acoger a una mujer casada sin la aprobación del marido puede suponer un atentado a la moral islámica, que tiene por objetivo la protección de la institución familiar. Finalmente, a esta realidad ayuda poco la apostilla final del primer párrafo del artículo 88.1 que estipula "la conciliación entre los esposos pondrá fin a la prohibición de contactar con la víctima", lo cual puede presuponer un clima de mayor presión y chantaje para la víctima por parte de entorno familiar en donde aún están muy consolidadas las estructuras patriarcales, fundamentadas siempre por componentes simbólico-religiosos de diversa naturaleza.

Además de lo citado anteriormente, otras aportaciones importantes de la Ley 103.13 en el ámbito penal están relacionadas con las siguientes cuestiones:

- El acoso sexual: la norma introduce ciertas mejoras relacionadas con el acoso sexual (al-taḥarruš al-ŷinsì). Anteriormente a la Ley 103-13, el Código Penal solo reconocía el acoso sexual cuando venía aparejado con el abuso de autoridad o el abuso de poder en el uso de su función, excluyendo otras posibles variantes o manifestaciones. Por su parte, la nueva norma (art. 503-1) no solo aumenta la pena de acoso por abuso de autoridad hasta los tres años de cárcel (anteriormente eran dos), sino que también introduce por vez primera el concepto de acoso sexual en los lugares públicos (actos, palabras o gestos de naturaleza o fin sexual) y de acoso sexual mediante mensajes escritos, telefónicos o electrónicos, grabaciones o imágenes que, en ambos casos, son castigados con una pena de cárcel de uno a seis meses y/o una multa de 2.000 a 10.000 dírhams. A este respecto Nuzha Skalli, parlamentaria del Partido del Progreso y el Socialismo (PPS) y antigua ministra durante el gobierno de Abbas el-Fassi (2007-2012), afirmaba que el artículo se encuentra a caballo entre la represión sexual y la lucha contra el acoso sexual, porque "no define el acoso sexual por el hecho de generar en la víctima un estado objetivo intimidante, hostil o degradante, tal y como lo había recomendado el Consejo Nacional de Derechos Humanos y, por último, tampoco identifica el sexo del acosador" (Skalli 2018 $)$. Es decir, este artículo se puede convertir en un instrumento que castiga a las personas que, de forma libre y voluntaria, deciden mantener relaciones sexuales 
fuera del marco institucional del matrimonio, cuestión ésta castigada por la moral islámica, como posteriormente contentaremos.

-El matrimonio forzado: la criminalización del matrimonio forzado es otra de las novedades de la Ley 103-13, que castiga con una pena de prisión de seis meses a un año y/o una multa de 10.000 a 30.000 dírhams a quien obligue a cualquier persona a contraer matrimonio mediante el uso de la violencia o las amenazas (art. 503-2-1). El hecho de especificar el recurso de la violencia y las amenazas es percibido por algunos actores sociales como una importante limitación de la ley, ya que pueden darse múltiples estrategias y factores para forzar a los hijos (especialmente a las hijas) al matrimonio sin hacer acopio de ello. Por otro lado, aunque es cierto que el párrafo segundo del art. 503-2-1 eleva al doble las penas en el caso de que la persona obligada a casarse sea una mujer por razón de sexo o una menor de edad o una mujer con discapacidad, sin embargo se especifica que "la acusación solo podrá realizarse mediante la denuncia de la persona lesionada", es decir, que deberían ser las propias mujeres en condiciones bastante precarias (sometidas a presiones familiares, niñas menores de edad, chicas limitadas por la diversidad funcional...) las que deben personarse en alguna instancia oficial para presentar denuncia. Finalmente tampoco ayuda a erradicar este grave problema social el último párrafo de este mismo artículo que señala que "la retirada de la denuncia pone fin al proceso judicial", ya que puede incitar a más presiones o coacciones del entorno familiar sobre las mujeres a fin de librar de las penas a los causantes del matrimonio forzado (padres, hermanos, tíos, etc.).

- Injurias y difamaciones: la Ley 103-13 introduce también como novedad los conceptos de injurias, difamación y atentado a la vida privada de las mujeres por razón de sexo (arts. 444-1 y 444-2), que son castigados con multas de 12.000 a 60.000 dirhams. En este sentido, se inserta un artículo específico (art. 447-3) mediante el cual se elevan las sanciones penales hasta cinco años de cárcel y hasta 50.000 dirhmas en el caso de interceptar, grabar o difundir palabras, informaciones, fotografías o vídeos de las mujeres, sin su consentimiento, por parte del marido, exmarido, novio, pareja, padre o tutor.

- La expulsión del domicilio conyugal. La nueva norma castiga la expulsión del hogar conyugal o la negativa a que retorne al hogar el cónyuge expulsado, elevando la pena hasta tres meses y una multa de 2.000 a 5.000 dírhams. Aunque se trata de una prescripción que puede beneficiar sobre todo a las mujeres (las esposas, principalmente) sin embargo resulta significativo que en este caso no se especifique la "razón de sexo", tan usual en otras casuísticas, y que de nuevo se recurra a coletilla de que "la 
retirada de la denuncia pone fin al proceso judicial" (art. 481-1), lo cual puede resultar muy perjudicial para la libertad y la autonomía personal de las mujeres víctimas. Como señala la Federación Internacional de Derechos Humanos: "tales disposiciones serían susceptibles de aumentar los riesgos para la víctima, que podría sufrir presiones o distintas formas de chantaje con el fin de obligarla a pedir la retirada de cargos" (FIDH 2018).

- La apropiación indebida de los bienes comunes. El nuevo texto castiga la disipación o la cesión de los bienes que se realiza de mala fe con la intención de dañar al otro cónyuge o a los hijos, o con la finalidad de eludir las responsabilidades relacionadas con la pensión alimenticia, el alojamiento o el reparto de bienes (art. 526-1). Este nuevo delito se castiga con pena de prisión de uno a seis meses y/o una multa de 2.000 a 10.000 dírhams. Aunque se trata de una norma que puede paliar, en alguna medida, la violencia económica en el ámbito conyugal, sin embargo resulta significativo que no se introduzca la noción de robo o hurto entre los esposos. Además, como en otras circunstancias mencionadas anteriormente, la denuncia debe realizarse obligatoriamente por el cónyuge perjudicado y, de nuevo, la retirada de la queja pone fin al proceso judicial y los efectos de la demanda (art. 526-1, párrafos segundo y tercero).

No obstante lo señalado anteriormente, la Ley 103-13 deja en el olvido algunas cuestiones importantes que habían sido reivindicadas por las asociaciones feministas y los organismos internacionales, como es el caso de la violación conyugal que, como tal, aparecía ya en el artículo 2 de la DEVM de 1993. El pensamiento, por tanto, que prevalece en esta Ley promulgada por el gobierno PJD es el tradicional "débito conyugal", fundamentado en el concepto de pecado de rebeldía de la esposa (nušūz) que aparece en el propio Corán ${ }^{13}$ y cuya lectura más literal castiga la negativa de la esposa a mantener relaciones con su marido con la amonestación, el aislamiento e, incluso, la violencia física. Por el contrario, las relaciones sexuales consentidas entre personas adultas no unidas por un contrato matrimonial siguen estando penalizas en Marruecos, ya que, a pesar de las reivindicaciones de la sociedad civil y las recomendaciones de diversos organismos, no ha sido modificado el artículo 490 del Código Penal que estipula penas de cárcel de un mes a un año. En este sentido, afirma Nouzha Skalli que "la filosofía del Código penal está fundamentada en conceptos moralizadores y obsoletos, como las buenas costumbres y el orden familiar" (Skalli 2018a.), lo cual supone ignorar las importantes transformaciones de la

${ }^{13}$ Corán 4:34 “Amonestad a aquellas (esposas) de quienes temáis que se rebelen (nuš̄uz), dejadlas solas en el lecho, pegadles. Si os obedecen, no os metáis más con ellas”. Trad. Julio Cortés. 
sociedad marroquí, especialmente la edad media de matrimonio, que en 2014 era de 31,3 años para los hombres y 25,7 para las mujeres (Haut-Commissariat au Plan 2016: 16). Aunque peor aún es el caso de las relaciones homosexuales, consideradas en el Código Penal como "perversión sexual" (al-šud $\underline{u} \underline{d} a l-\hat{y} i n s \bar{l})$, que siguen estando castigadas con penas de cárcel de seis meses a tres años y multa de 200 a 1000 dirhams (art. 489).

\section{La polarización del debate sobre la Ley $n^{0} 103.13$}

Como dijimos anteriormente, el debate se polariza en dos modelos de sociedad que parecen antagónicos cuando el tema se focaliza en las mujeres, la familia, la sexualidad, etc. Por un lado, las tendencias cuyos referentes ideológicos se encuentran anclados en los denominados "referentes universales" (Declaración Universal de Derechos Humanos, CEDAW, Plataforma de Beijin, etc.) y que, en el tema que nos ocupa, parten del concepto "género" como una construcción sociocultural de naturaleza patriarcal, basada en unas dinámicas de poder y de conflicto, que favorece la discriminación de las mujeres y cuya consecuencia, entre otras, es la violencia de género. Aunque se trata de una tendencia plural y diversa, entre la que podríamos ubicar a las asociaciones feministas y de derechos humanos, a la sociedad civil laica, partidos de izquierdas, etc., de estos movimientos parte la defensa de un modelo de sociedad de corte laico, en estrecha colaboración con las instancias internaciones y las organizaciones extranjeras.

En el polo opuesto estarían las corrientes que "abordan la cuestión de la mujer desde la perspectiva moral de la revelación que aportan sus fuentes, principalmente Corán y sunna, y desde la revisión analítica de la historia social del Islam" (Macías 2005: 104-105), entre las cuales podríamos ubicar a los partidos y movimientos islamistas, las instituciones islámicas gubernamentales y no gubernamentales, asociaciones de ulemas, adules y alfaquíes, ciertos movimientos de protección de la familia, etc. Aunque también se trata de un movimiento heterogéneo, para la mayoría de ellos la secularización es percibida como una amenaza, como algo extraño a la cultura musulmana, como antesala de la degradación moral y como una invasión neo-imperialista orquestada desde organismos internacionales o países extranjeros. En el caso concreto que nos ocupa, el PJD y su alter ego, el Movimiento de la Unidad y la Reforma (MUR), consideran ${ }^{14}$ que los presupuestos laicos y el enfoque de género que pretenden imponer los agentes occidentales son anti-islámicos, en el sentido de

${ }^{14}$ El pensamiento del MUR a propósito de las mujeres y la familia está recogido en el informe que realizó en el año 2000 para posicionarse en contra del Plan de Acción Nacional para la Integración de la Mujer al Desarrollo (MUR, 2000). Dicho informe se encuentra analizado en Pérez y Macías (2017: 16-18).

Araucaria. Revista Iberoamericana de Filosofia, Política, Humanidades y Relaciones Internacionales, año $23, \mathrm{n}^{\circ} 48$. Tercer cuatrimestre de 2021. Pp. 165-185. ISSN 1575-6823 e-ISSN 2340-2199 https://dx.doi.org/10.12795/araucaria.2021.i48.08 
que no respetan los principios ni las normas de la $\check{s} a r \bar{\imath} ` a$, y por tanto, suponen un peligro para la estabilidad social y la idiosincrasia de la familia musulmana, que debe estar basada en relaciones de complementariedad, así como para la propia cultura nacional de Marruecos (MUR 2000).

En el marco de este debate enconado, las principales voces que salen a la palestra para defender a ultranza el contenido de la Ley 103-13 proceden de las élites políticas del PJD, empezando por la propia ministra de la Familia, Basima Haqawi, quien afirma taxativamente:

Se trata de uno de los textos más importantes que refuerzan el arsenal jurídico nacional en materia de igualdad entre los sexos, junto con la Mudawwana (Ley de la Familia), que ha creado una revolución en este ámbito (...). Un verdadero salto cualitativo en el proceso de promoción de la condición de la mujer marroquí, porque adopta un enfoque legal que no se basa únicamente en medidas represivas (HuffPost Maroc 2018).

Contrariamente a lo que opinan sus detractores, la ministra Haqawi considera que la Ley 103-13 responde plenamente a las expectativas de las mujeres, porque no solamente reposa sobre medidas represivas y coercitivas, sino que tiene además dos ventajas añadidas: en primer lugar, ha reforzado la atención a las mujeres víctimas de violencia dentro de las instituciones del país, tanto a nivel nacional como regional y local; en segundo lugar, según palabras de la ministra, la ley "ha abierto el camino para que la sociedad civil pueda aportar su contribución e implicarse en ello" (HuffPost Maroc 2018). No obstante, como señalaremos posteriormente, varias asociaciones han denunciado su exclusión en el proceso de formulación y adopción de la Ley. .

Definido por ella misma como "texto de referencia" (Lahrache, 2018), Basima Haqawi concluye que la Ley 103-13 se enmarca dentro de los principios de equidad y justicia que establece la Constitución de 2011, especialmente sus artículos consagrados a la igualdad de derechos civiles, políticos, económicos y medioambientales entre hombres y mujeres (art. 19), el derecho a la seguridad de las personas (art. 21) y el derecho a la integridad física y moral que presupone la prohibición del trato cruel, inhumano y degradante (art. 22):

Se trata de un texto fundamental que comprende la definición de conceptos, disposiciones penales, medidas preventivas y iniciativas de protección, así como un mecanismo institucional integrado de apoyo mediante el endurecimiento de las penas contra los responsables. El texto se inscribe en el marco de la consolidación de la democracia y la realización de la paridad y de sus valores de justicia, conforme a las disposiciones de la Constitución del 2011 (Lahchare 2018). 
Muy próxima a la opinión de la ministra se encuentra la del resto de parlamentarios del PJD, entre los cuales se ha manifestado de forma especial Boutaina Karouri, miembro de la Comisión parlamentaria de justicia, legislación y derechos humanos, quien afirma que la Ley supone "un verdadero salto cualitativo en el proceso de promoción de la mujer marroquí" (MAP 2018) y, contrariamente a quienes opinan que la Ley 103-13 ha obviado manifestaciones de violencia tan usuales como la que sufren las mujeres en el ámbito conyugal, Karouri considera que la definición de violencia contra la mujer que aporta la norma es suficiente, puesto que engloba todas las formas posibles.

En cuanto al sector crítico, este incide particularmente en la exclusión de la Ley de la mayor parte de las reivindicaciones de la sociedad civil que viene trabajando sobre el terreno desde hace décadas, la cual se ha sentido discriminada del proceso llevado a cabo por el PJD, "a pesar de su incesante combate para una legislación de calidad en materia de lucha contra la violencia hacia las mujeres" (Skalli 2018a). En este sentido, poco después de la aprobación de la Ley en el parlamento, la Federación Internacional de Derechos Humanos emitió un comunicado en el que, además de demostrar su decepción con el resultado final, denunciada también la exclusión de la sociedad civil que hemos señalado:

La ley marroquí contra la violencia contra las mujeres, aprobada el 14 de febrero, es una reforma conservadora, cosmética e insuficiente para combatir el azote de la violencia contra las mujeres y constituye una oportunidad perdida para proteger plenamente los derechos de las mujeres marroquíes. A pesar de años de discusiones, nuestras organizaciones denuncian la falta de una consulta real con la sociedad civil y el hecho de no tener en cuenta la mayoría de sus recomendaciones, particularmente en lo que respecta a la penalización de la violación conyugal (FIDH, 2018).

De igual forma, este sector le ha reprochado al PJD que no haya tenido en cuenta las recomendaciones formuladas desde organismos nacionales, principalmente el Consejo Nacional de Derechos Humanos, muy implicado en este asunto, e internacionales como ONU-Mujer, Amnistía Internacional o la propia Federación Internacional de Derechos Humanos, de tal manera que el texto resultante, condicionado por cuestiones conservadoras y moralizantes, no tiene en cuenta las convenciones internacionales que han sido ratificadas por Marruecos, ni tampoco las prescripciones igualitarias establecidas en la Constitución de 2011. En este sentido afirmaba Atefa Timjerdine, miembro de la Coalición al-Karama, que la Ley no solamente supone una regresión con respecto a otros proyectos de ley anteriores, sino también "una violación del texto constitucional, que no conducirá a la eliminación de la violencia que sufren las mujeres marroquíes" (MAP, 2018), es decir, una visión diametralmente 
contraria a las opiniones vertidas por las líderes del PJD, como hemos señalado anteriormente.

En esta misma línea de pensamiento se encuentra la opinión de Nouzha Skalli, parlamentaria por el Partido del Progreso y de Socialismo y afamada feminista del país, quien afirma:

La ley 103-13 no cumple con las expectativas de las asociaciones a favor de derechos de las mujeres. Es una ley que carece de preámbulo para anunciar sus objetivos. No penaliza ni el robo entre cónyuges ni la violación conyugal y se integra perfectamente en el espíritu obsoleto y moralizador del Código Penal, más enfocado en la preservación de las buenas costumbres y el orden familiar que en la protección física y moral de las mujeres (Skalli 2018b).

En el mismo sentido, Saida Drissi Amrani, presidenta de la Asociación Democrática de Mujeres de Marruecos (sede de Rabat), mostraba públicamente su decepción ante una ley que ella considera vacía y que se limita a modificar parcialmente el Código Penal, argumentando además: "las definiciones de las infracciones están muy limitadas y la cuestión de la prevención, la atención y la protección de la víctima, así como la reparación de daños, no están a la altura" (Pigaglio ${ }^{2018)}$, para concluir diciendo: "Marruecos no está a la altura de la dramática situación de la violencia contra las mujeres en el país; estamos muy por debajo de la ley integral conseguida por las feministas tunecinas el pasado verano" (FIDH, 2018).

Desde el punto de vista político, las críticas se focalizan en la ministra que ha llevado la voz cantante durante todo el proceso de redacción, revisión y adopción definitiva de la Ley 103-13, Basima Haqawi, quien "contando con el apoyo del sector femenino del islamismo marroquí, como el Forum Ezzahrae, defienden un modelo singular de mujeres cisgénero, indisociable del núcleo familiar en el marco del referencial religioso" (Kheireddine 2018), como opina Aïda Kheireddine, de la Fondation Heinrich-Böll-Stiftung. Pero igualmente las críticas se prolongan al pensamiento conservador que domina en todo el gobierno del PJD y a la "persistencia de mentalidades retrógradas y misóginas en el parlamento" (Filoche 2018), tal y como lo expresa en un comunicado el Movimiento Alternativo para las Libertades Individuales de Marruecos. En el ámbito parlamentario, Nuzha Skalli se lamenta de que esta ley haya sido una ocasión perdida para alcanzar una unanimidad en el tema de la protección de las mujeres contra la violencia, por encima de cualquier diferencia ideológica, partidista o identitaria, concluyendo que "era demasiado esperar de un gobierno dirigido por un partido conservador apegado a un referencial moralizador y patriarcal" (Skalli 2018a). 


\section{A modo de conclusión}

Reivindicada durante décadas, la Ley de violencia contra las mujeres traduce, en buena medida, las dinámicas ideológicas de la sociedad marroquí, cuyos modelos de sociedad parecen antagónicos cuando el foco de atención son las mujeres y el prototipo familiar que debe acompañar al cambio social. Si los referentes ideológicos de la sociedad civil más comprometida con la violencia de género se hallan ubicados en el marco de los instrumentos internacionales más vanguardistas en la materia señalada (DEVM, CEDAW, Declaración de Beijing, Convenio de Estambul), por su parte, el modelo islamo-islamista, en el que se ubica el gobierno del PJD, parece cuestionar estas narrativas globalizantes de carácter laico, a las que siempre considera eurocéntricas, impositivas y anti-islámicas. Frete a ello, y, como praxis contrahegemónica, la tendencia islamo-islamista busca espacios de autonomía cultural e ideológica y propone una interpretación/adaptación de ciertas especificidades religiosas, históricas y nacionales, que parten de la preponderancia (dominación) del grupo familiar sobre los derechos fundamentales de los miembros que la componen, especialmente de las mujeres. Dentro de este pensamiento, se llega a castigar cualquier manifestación pública o privada que trasgreda los límites de la moral islámica, al tiempo que se legitiman ciertas acciones violentas contra las mujeres como medida de corrección, advertencia o punición por faltas que se consideran que traspasan las fronteras de las buenas costumbres y que podrían poner en riesgo el equilibrio de la familia.

Entre otras consecuencias, este enfrentamiento ideológico provocó que la ley sobre violencia contra las mujeres conociera varios anteproyectos desde el año 2003 y que el último de ellos (Ley 103.13), bastante más moderado, tardara seis años en ser aprobado definitivamente por el parlamento, con mayoría del PJD, contando además con el malestar de los partidos de izquierda, de las ONG's relacionadas con el tema y de organismos oficiales de carácter independiente, como el Consejo Nacional de Derechos Humanos, que durante todo estos años habían formulado importantes recomendaciones y propuestas, en el marco de los referentes internacionales anteriormente señalados, que no fueron tenidas en cuenta en la medida que ellos pretendían. De tal forma que cuestiones tan importantes como la violencia conyugal, la violación conyugal, las medidas cautelares urgentes para las mujeres víctimas, el establecimiento de recursos de protección y acogida y la despenalización de las relaciones sexuales consentidas entre adultos no casados (hetero u homosexuales), entre otras cuestiones, han quedado en el tintero a la espera de tiempos mejores. En otros casos, los logros obtenidos se ven empañados por la introducción de fórmulas que pueden actuar en contra de las propias mujeres víctimas de violencia, como es el caso de que la conciliación de los esposos o la retirada de 
las denuncias pongan fin a los procedimientos judiciales. Como han señalado los grupos opuestos a la Ley, estas apostillas puede suponer para las mujeres víctimas mayores presiones, coacciones o chantajes por parte del entorno familiar de ella o del esposo/exesposo/pareja.

No obstante y, a pesar de la polarización ideológica señalada, Ley $\mathrm{n}^{\mathrm{o}}$ 103.13 traduce también el pragmatismo del gobierno del PJD, ya que ha logrado oficializar del tema de la violencia de género en manos de su ministra más carismática, Basima Haqawi, y en definitiva, ha sacado a la luz una Ley de violencia contra las mujeres que tiene por objetivo responder a los compromisos y exigencias adquiridos por el estado marroquí a nivel nacional (Constitución, reivindicaciones de la sociedad civil) y, sobre todo, a nivel internacional (Declaración de Beijing, CEDAW, etc.). Ciertamente no se trata de objetivos ambiciosos, como reclamaba buena parte de la sociedad civil del país, sino más bien de compromisos mínimos que posiblemente resultarán insuficientes para defender los derechos de las mujeres que, por el hecho de serlo, sufren diversas formas de violencia que atentan contra sus derechos fundamentales.

\section{Bibliografía:}

Al-Ŷarīda al-Rasmiyya 2018, "Zahīr šarīf raqm 1.18 .19 șādir fī 5 ŷumādà alājira 1439 (22 fibrāyir 2018) bi-tanfịd al-Qānūn raqam 103.13 al-muta'alliq bi-muḥāraba al- 'unf ḍidd al-nisā'”' en Al-Ŷarìda al-Rasmiyya, al-Mamlaka al-Magribiyya, 6655 (23 ŷumādà al-ājira 1439 / 12 de marzo), pp. 14491455.

AMVEF 2010, L'hébergement dans le processus d'empowerment des femmes victimes de violence: Concepts, enjeux et défis, Casablanca.

Benabdallah, M.A. 2012. "Le Bulletin officiel et la production normative» en Revue Marocaine d'Administration Locale et de Développement, 107, pp. 359-363.

Bulletin Officiel 2014, "Dahir $\mathrm{n}^{\circ}$ 1-14-06 rabii II 1435 portant promulgation de la Loi n ${ }^{\circ}$ 15-14 modifiant et complétant l'article 475 du Code pénal" en Bulletin Officiel du Royaume du Maroc, 6240 (18 joumada I 1435 / 20 mars), 2492.

Bulletin Officiel 2018, "Dahir no 1-18-15 du 5 joumada II 1439 (22 fávrier 2018) portant promulgation de la loi $\mathrm{n}^{\circ} 103-13$ relative à la lutte contre les violences faites aux femmes" en Bulletin Officiel du Royaume du Maroc, 6688 (21 chaoual 1439 / 5 juillet), pp. 1384-1389.

Conseil Economique, Social et Environnemental 2006, Les dimensions sociales de l'égalité entre les femmes et les hommes. Constats et Recommandations, Rabat. 
Conseil National des Droits de l'Homme 2018, Avis du Conseil National des Droits de l'Homme à propos du Projet de Loi $n^{\circ} 103.3$ relatif à la lutte contre la violence à l'égard des femmes, https://www.cndh.org.ma/sites/ default/files/avis violence_a legard des_femmes francais.pdf [13-102021].

Chikhaoui, N. 2008, Étude d'évaluation de l'impact des campagnes nationales de lutte contre la violence fondée sur le genre, Rabat.

Desrues, T. y Fernández, I. 2013, "L’expérience gouvernementale du Parti de la Justice et du Développement : les islamistes au pouvoir ?" en L'Année du Maghreb, 9, pp. 345-365. Doi: https://doi.org/10.4000/ anneemaghreb.1954.

Desrues, T. y Hernando de Larramendi, M. 2011, "La gobernanza de Mohamed VI: legado autoritario, léxico democrático, pedagogía participativa e inserción internacional" [en T. Desrues y M. Hernando Larramendi, eds.: Mohamed VI. Política y cambio social, Córdoba, Almuzara], pp. 21-93.

Dialmy, A. 2012, Programme multisectoriel de lutte contre les violences fondées sur le genre par l'autonomisation des femmes et des filles -Tamkine-, Rabat.

FIDH 2018, "Loi marocaine contre les violences faites aux femmes : quand la montagne accouche d'une souris", https:/www.fidh.org/fr/regions/ maghreb-moyen-orient/maroc/loi-marocaine-contre-les-violences-faitesaux-femmes-quand-la [13-10-2021].

Filoche, A, 2018, "La loi contre les violences faites aux femmes divise le Maroc ", https://lepetitjournal.com/casablanca/actualites/la-loi-contreles-violences-faites-aux-femmes-divise-le-maroc-223759 [13-01-2021].

Haut-Commissariat au Plan 2012, Enquête nationale sur la prévalence de la violence à l'égard des femmes au Maroc, Rabat.

Haut-Commissariat au Plan 2016, La femme marocaine en chiffres. Tendances d'évolution des caractéristiques démographiques, Rabat.

Haut-Commissariat au Plan 2019, Rapport sur les violences faites aux femmes et aux filles, Rabat.

HuffPost Maroc 2018, "La Loi 103-13 contre les violences faites aux femmes entre (enfin) en vigueur" en Monasso (13-09-2018), https://monasso. ma/134845-la-loi-103-13-contre-les-violences-faites-aux-femmes-entreenfin-en-vigueur [16-11-2020].

Human Rights Watch 2018, "Maroc: une nouvelle loi contre les violences faites aux femmes", https:/www.hrw.org/fr/news/2018/02/26/maroc-unenouvelle-loi-contre-les-violences-faites-aux-femmes [13-10-2021].

Jeddi, D. 2015, "La violencia conyugal en el Código Penal de Marruecos" en Revista de Derecho UNED, 16, pp. 1007-1033. 
Kheireddine, A. 2018, "Pourquoi la nouvelle loi marocaine contre la violence faite aux femmes n'est pas suffisante pour changer le status quo", https:// ma.boell.org/fr/2018/04/13/pourquoi-la-nouvelle-loi-marocaine-contrela-violence-faite-aux-femmes-nest-pas [13-10-2021].

Lahrache, A. 2018, "Adoptée à la majorité par la Chambre des représentants à la mi-février dernier", https://lematin.ma/journal/2018/loi-relative-luttecontre-violence-faite-aux-femmes-entre-vigueur/300519.html $\quad[13-10$ 2021].

Lefébure, A. 2016, "Adoption du projet de loi sur les violences faites aux femmes: Les députés masculins ont déserté les discussions" en HuffPost Maroc, https://www.huffpostmaghreb.com/2016/07/21/violencesfemmes-adoption-projet-loi-deputes-masculins_n_11114716.html [10-112020].

Lefébure, A. 2020, "Confinement : une baisse des violences faites aux femmes, vraiment?" en Telquel, (2-05-2020), https:/telquel.ma/2020/05/06/ confinement-une-baisse-des-violences-faites-aux-femmesvraiment 1682624 [14-11-2020].

MAP 2018, "La loi relative à la lutte contre la violence faite aux femmes à l'épreuve" https://www.maroc.ma/fr/actualites/la-loi-relative-la-luttecontre-la-violence-faite-aux-femmes-lepreuve [13-10-2021].

MAP 2020, "Confinement: dix fois moins de poursuites pour violences faites aux femmes par rapport à la moyenne mensuelle", https://www.maroc.ma/ fr/actualites/confinement-dix-fois-moins-de-poursuites-pour-violencesfaites-aux-femmes-par-rapport-la [13-10-2021].

Macías, J.A. 2005, "La mujer en la ideología del movimiento islamista de Marruecos" en Miscelánea de Estudios Árabes y Hebraicos. Sección Árabe Islam 54, pp. 101-115.

MUR 2000, Mawqifu-nā mimmā summiya Mašrū' juțtat al- 'amal al-wațaniyya li-idmāŷy al-mar'a fì l-tanmiyya, Casablanca, Manšūrāt al-Furqān.

Nima, M. 2018, “The Victimization of the 'Muslim Woman': The Case of Amina Filali, Morocco" en Journal of International Women's Studies, 19, 3, pp. 49-59.

Olmedo, C. 2006. "Asociacionismo femenino en Marruecos. Un estudio histórico-antropológico" [en C. Pérez, ed.: Sociedad civil, derechos humanos y democracia en Marruecos, Granada, Universidad], pp. 267293.

ONU Mujeres 2010, Manuel de legislación sobre la violencia contra la mujer, https://www.un.org/womenwatch/daw/vaw/handbook/Handbook-forlegislation-on-VAW-(Spanish).pdf [13-10-2021]. 
Parejo, M.A. 2010, "Los sonoros silencios de la reforma constitucional en el Marruecos de Mohamed VI" [en M.A. Parejo (ed.): Entre el autoritarismo y la democracia. Los procesos electorales en el Magreb, Barcelona, Bellaterra], pp. 365-412.

Pérez, C. 2020, "La violencia de género en Marruecos: una aproximación a la realidad social y al debate ideológico" en Investigaciones Feministas, 11, 2, pp. 343-353, https://doi.org/10.5209/infe.65363.

Pérez, C. y Macías, J.A. 2017, "La construcción histórica de la hegemonía ideológica del PJD en Marruecos: la marcha islámica de 2000 y el debate sobre las mujeres" en Revista de Estudios Internacionales Mediterráneos, 22, pp. 1-34, https://doi.org/10.15366/reim2017.22.001.

Pigaglio R. 2018, "Le Maroc adopte une loi contre les violences faites aux femmes" en La Croix, https://www.la-croix.com/Monde/Afrique/Le-Maroc-adopteloi-contre-violences-faites-femmes-2018-02-15-1200914092 [13-102021].

Secrétariat d'Etat Chargé de la Famille 2015, Plan opérationnel de la stratégie nationale de lutte contre la violence à l'égard des femmes, Rabat.

Skalli, N. 2018a, "Le Maroc mérite mieux que la nouvelle loi contre la violence envers les femmes", https://www.tanmia.ma/slideshow/02/28/21388/ [1310-2021].

Skalli, N. 2018b, "Non! La loi 103-13 ne protège pas les femmes ", Tribune, 5220, https://www.leconomiste.com/article/1024751-non-la-loi-103-13ne-protege-pas-les-femmes [13-10-2021].

White, G. W. 2008, "El 'fin de la era de la laxitud' en Marruecos" [en Y. Zoubir y H. Amirah, eds.: El Magreb. Realidades nacionales y dinámicas regionales, Madrid], pp. 107-126.

Zirari, M. 2007, Plaidoyer pour une nouvelle approche du Code Pénal. Pour le respect des droits fondamentaux des femmes, Casablanca. 
